\title{
L’interaction Micro-Assurances Et Assurés Dans Le Processus De Couverture Maladie En Côte d'Ivoire
}

\author{
Fofana Memon \\ Université Peleforo Gon Coulibaly, Korhogo, Côte d'Ivoire \\ Kouame Komenan \\ Université Félix Houphouët-Boigny-Abidjan
}

doi: 10.19044/esj.2016.v12n13p468 URL:http://dx.doi.org/10.19044/esj.2016.v12n13p468

\begin{abstract}
The micro health insurance have greatly contributed to the medical coverage of the Ivorian population. This text is to question the social behavior of micro-insurance and insured to explain how they disrupt the disease process coverage of the individual. In terms of methodology, this article is based on a qualitative approach based on direct and indirect observation, document review and interviews. The results of the study indicate that microinsurance disease involved, certainly, to improve access of the population to health but the behavior of the insurer and the insured may hinder access to care health. This is the adverse selection behavior that the study seen at the micro-insurance and skilled behavior of moral hazard at the insured.
\end{abstract}

Keywords: Healthcare coverage, Microinsurance, Social Assured, Ivory Coast.

\section{Résumé}

Depuis les années 1970, les micro-assurances maladie ont fortement contribués à la couverture médicale de la population ivoirienne. Le présent texte vise à questionner les comportements sociaux des micro-assurances et des assurés sociaux afin d'expliquer la façon dont ces acteurs perturbent le processus de couverture maladie de l'individu. Au plan méthodologique, cet article repose sur une approche qualitative axée sur l'observation directe et indirecte, la revue documentaire et des entretiens. Les résultats de l'étude indiquent que les micro-assurances maladie participent, certes, à l'amélioration de l'accès de la population aux soins de santé mais certains comportements de l'assureur et de l'assuré peuvent freiner l'accès à ces soins. Il s'agit principalement du comportement d'anti-sélection que l'étude 
à pu constater au niveau des micro-assurances et du comportement que nous qualifions d'aléa morale au niveau de l’assuré.

Mots clés : Couverture maladie, Micro-assurance, Assurés sociaux, Côte d'Ivoire.

\section{Introduction :}

L’accès aux soins de santé est un droit humain fondamental (Patrick. et al, 2004). Certains travaux (Patrick et al, 2004 ; N'guessan. \& Bouaffon, 2006), constatent que cet accès est assez limité à une grande partie de la population. En Côte d'Ivoire, les décennies 1960 et 1970 ont été marquées par l'accès gratuit aux soins de santé (DIPE, 2008). Le financement reposait sur le budget de l'Etat. Cependant, à partir des années 80, l'une des difficultés sanitaires concerne la couverture sanitaire très disparate sur l'étendue du territoire. Ainsi, la politique de gratuité à cette époque s'est avérée non seulement assez onéreuse pour les finances publiques. En outre, elle a été porteuse d'un déséquilibre entre les zones urbaines et les milieux ruraux. L’option de gratuité a été progressivement abandonnée après 1980 (DFR, CNS, DIPE, 2007) par L’Etat qui a décidé que la population contribue désormais aux dépenses de leur propre santé. Les recettes ainsi recouvrées constituent les ressources propres pour les établissements sanitaires.

Cette politique contributive aux frais sanitaires et médicamenteux par la population ne favorise toujours pas une couverture maladie pour les catégories sociales pauvres qui représentent d'ailleurs la plus grande partie de la population ivoirienne. Malgré l'avènement d'alternatives comme les institutions de micro-assurance maladie, des comportements peuvent constituer un frein ou une source d’inégalité sociale dans la viabilité de l'institution d'assurance étant donné que le principe est la solidarité caractérisée par le paiement d'une cotisation et la gestion collective des risques maladies. Dans le jeu de rapport, l'assuré a pour objectif la maximisation de ses soins de santé et la minimisation des coûts de soins prescrits. Par contre, l'assureur veille à ce que l'assuré soit satisfait en lui proposant une prime d'assurance. Or sur le marché médical, certains clients présentent un grand risque de maladie par rapport à d'autres. Des assurés présentant de faibles risques ont tendance à payer des primes élevées pour une petite couverture. Ce constat permet d'émettre l'hypothèse selon laquelle les comportements d'anti-sélection ${ }^{9}$ et d'aléa moral sont de natures à favoriser l'inégalité dans le processus de couverture maladie au sein de la population et d'affaiblir aussi la durabilité des entreprises de micro-

${ }^{9}$ C'est lorsque les maison de micro-assurance souhaite avoir des assuré qui ne présente pas trop de problème de santé ou qui consomme moins de soins. 
assurance. Comment les comportements d'anti-sélection et d'aléa moral constituent-ils une menace à la viabilité de l'institution micro-assurance et une source de perturbation de la couverture médicale de l'usager? Ce présent article répond à cette question en présentant (i) la méthodologie de l'étude, (ii) le lien entre le comportement d'anti-sélection et inégalité au niveau de la couverture maladie, (iii) le lien entre aléa morale et couverture maladie.

\section{Méthodologie de l'étude}

\subsection{Approche de l'étude, acteurs cibles et technique de collecte des données}

La présente étude est basée sur une approche qualitative à visée compréhensive axée sur les entretiens semi-structurés, des observations, des focus et des données secondaires. L'approche qualitative de terrain a été adoptée en privilégiant la «biographie thérapeutique» des assurés qui a été réalisée à travers des observations directes et des entretiens individuels auprès de 67 acteurs dont la plupart ont expérimenté la relation avec des micro-assurances maladie. Les entretiens se sont déroulés auprès de deux micro-assurances installées dans le district d'Abidjan où une soixantaine d'entretiens individuels ont été réalisés sur les thèmes prioritaires de l'étude à savoir (i) le fonctionnement et les dynamiques des institutions de microassurance maladie; (ii) la modalité de souscription à une assurance et leurs implications sur la durabilité de l'accord assureur-assuré. Des entretiens individuels réunissaient de façons spontanées en moyenne quatre personnes. Ces types d'entretien pour plus d'intensité productive de données empiriques. Les entretiens ont été effectés avec dix (10) assureurs, et cinquante-sept (57) assurés. La méthode de sélection a été possible grâce à la technique de réseau dans la mesure où les assurés sont 'invisibles'. Les acteurs enquêtés ont été classés en deux groupes. Chez les assureurs, l'étude a distingué les responsables et les agents. Au niveau des assurés, c'est-à-dire les usagers qui ont souscrit à une maison d'assurance, l'interview a été réalisé avec ceux qui ont souscrit il y’a plus de cinq ou dix années et ceux qui ont une adhésion récente, c'est-à-dire moins de cinq ans à la maison d'assurance. Le comportement d'anti-sélection et d'aléa moral ont été saisis à travers les propos et les comportements des acteurs réels. De ce point de vue, l'étude s'est inscrite dans la perspective du populisme et de l'individualisme méthodologiques en tant que démarche intellectuelle privilégiant la compréhension des attitudes et des comportements des acteurs sociaux (Olivier de Sardan, 2000). La méthode d'analyse des données est basée sur l'analyse de contenu en rapport avec les thématiques prioritaires de l'étude. 


\subsection{Anonymisation et analyse des données}

Le recueil des données surtout avec les fournisseurs de prestation s'est déroulé sur plus de neuf mois, c'est-à-dire de décembre 2014 à septembre 2015. L'accord de participation à l'étude des différents fournisseurs de prestation n'a pas été une tâche aisée. Des efforts importants ont été nécessaires pour convaincre les partenaires concernés de participer à la présente étude. L'anonymat du nom des institutions visitées, des personnes enquêtées et la protection des données a été la première condition d'attraction des acteurs cibles à participer aux échanges. Ensuite, la mobilisation du réseau a permis de pénétrer le système et de recueillir des données avec la confiance des acteurs, qu'il s’agisse des assurés ou des fournisseurs de prestations. Ainsi, pour garantir l'anonymat des usagers et des maisons d'assurances qui ont fait l'objet d'enquête, les données ont été cryptées à travers le discours «les noms, prénoms et adresses ne nous intéresses pas ». L'anonymisation s'est imposée pour le recueillement et l'analyse des données surtout du côté des assureurs ou fournisseurs des prestations. Sur cette base, l'étude n'a pas voulu cité le nom des maisons d'assurances explorées à cet effet. Ce qui est sans doute rassurant pour la protection avec efficacité les données, qu'il s’agisse des assurés ou des fournisseurs de prestations.

2. Comportement anti-sélection, aléa moral et inégalité de couverture du risque maladie par les micro-assurances de santé

o Cadre juridique réglementant le métier de micro-assurance maladie en Côte d'Ivoire.

Plusieurs lois et décrets règlementent le secteur de l'assurance en Côte d'Ivoire depuis l'indépendance. En effet, La loi n 60-342 du 28 octobre 1960 et son décret d'application n ${ }^{\circ}$ 61-370 du 13 novembre 1961 instituent l'obligation d'assurance automobile en 1962. La lois n ${ }^{\circ} 62-232 \mathrm{du}$ 29 juin 1962, règlemente l'assurance et organise la profession d'assureurs.

En 1989, pour remédier aux difficultés de l'assurance automobile du fait du niveau des indemnités, la loi n89-1301 du 18 décembre 1989 et son décret d'application $n^{\circ}$ 90-89 du 17 janvier 1989 déterminant les préjudices indemnisable et fixent un barème d'indemnisation.

La loi $\mathrm{n}^{\circ}$ 86-485 du 1er juillet 1986 rendant obligatoire la domiciliation locale des facultés à l'importation a été abrogée par l'ordonnance $\mathrm{n}^{\circ} 97-444$ du 08 aout 1997. Ainsi, la domiciliation de l'assurance des facultés à l'importation est rendue obligatoire par l'ordonnance $n^{\circ}$ 2007-478 du 16 mai 2007.

Dans les années 90, le pays comptait une quarantaine de sociétés d'assurances (Yéo Sana, 2012). La politique d'assainissement mis en place 
par les autorités a permis de réduire ce nombre à une vingtaine. Avec les nouveaux agréments octroyés par la suite, le nombre de société d'assurance opérant sur le marché ivoirien, en 2007, était de trente-deux (32) dont onze (11) sociétés d'assurance-vie et vingt et une (21) société d'assurance-IARD (Incendie, Accidents et Risque Divers). Ces sociétés sont accompagnées dans leurs activités par des intermédiaires dont une soixantaine de coutiers ou sociétés de courtage en assurance. La présente étude porte sur les microassurances, c’est-à-dire les petites structures d'assurance qui exercent dans la capitale économique du pays.

\subsection{Comportement anti-sélection, divinisation de la santé et inégalité d'accès aux soins}

L’analyse des entretiens réalisés laisse apparaître la réalité sociale de divinisation que les maisons de micro-assurance réservent à leur usager ou aux assurés. En effet, les assureurs ou fournisseurs de prestation de service de micro-assurance demandent aux usagers 'd'éviter de tomber malades" et de se protéger contre les endémies et/ou maladies graves. Les sociétés de micro-assurance demandent donc aux assurés de ne plus présenter de risques de maladies graves et/ou chroniques. Cet enquêté ne dit pas autre chose lorsqu'il affirme :

«Quand j'ai souscrit à leur assurance, ils m'ont dit, après avoir fini tout le processus de souscription, qu'ils ne prennent pas en charge les endémies et autres maladies jugées très graves; Je leur ai demandé si je suis Dieu pour savoir ce qui va m'arriver demain ? Et même quand ma fille est tombée malade sur une année, après quelques mois de prise en charge, ils m'ont dit que comme la maladie ne finit pas, l'assurance ne pourra plus prendre en charge les frais ».

Des propos précédant, comment l'assuré peut-il 's's'abstenir' des endémies ou des maladies graves/chroniques ? Mais comment peut-il accepter d'être atteint d'une maladie jugée grave ou mineure ? Les microassurances demandent alors aux assurés de prendre toutes les mesures de prévention utile et d'avoir les ressources nécessaires pour éviter de contracter des endémies et/ou des maladies graves. Les assurés interrogés, sont très diversifiés et homogènes quant à la demande impossible qui structure les relations qu'ils entretiennent avec les assureurs derrière la formule :

«Ils ne se comportent pas en humain, ils nous demandent d'être des dieux en nous demandant de nous abstenir des graves maladies ».

Les entretiens ont revélé que la durée de la relation ou du contrat assurés-assureurs est adossé à la nature de la maladie que l'assuré présentera un jour ou l'autre à l'assureurs et pour laquelle il demande une prise en charge des frais médicamenteux. Car le contrat risque d'être interrompu parce que l'assuré n’a pas eu gain de cause du fait de la maladie jugée grave 
ou inguérissable par la micro-assurance. Cette thèse est soutenue par le discours d'un agent d'assurance en ces termes "une seule assurance n'accompagne pas son client jusqu'à sa tombe ». A l'analyse, cette formule de “divinisation” de la santé que les assureurs demandent aux assurés ne fait que produire et renforcer l’inégalité d’accès aux soins.

En effet, les assurés atteints d'une maladie jugée grave et en situation de faible dotation économique ne pourront plus bénéficier d'une couverture maladie. C'est donc le fait de refus des assureurs de couvrir certaines pathologies qui freine l'évolution du taux de couverture assurance maladie. Cette analyse est corroborée par les données de l'Enquête Démographique et de Santé (EDS), 2012, : «la majorité des hommes et des femmes enquêtés n’ont aucune assurance médicale. En effet les pourcentages des femmes et des hommes ne disposant pas d'assurance médicale sont respectivement de 97 \% et 94 \%. Quelles que soient les caractéristiques sociodémographiques des femmes et des hommes, ces proportions sont très élevées. Le type d'assurance auquel les femmes et les hommes ont le plus recours est l'assurance mutuelle ou l'assurance de santé communautaire (2\% pour les femmes et $3 \%$ pour les hommes). Seulement, $1 \%$ des femmes et $2 \%$ des hommes sont assurés par leur employeur. Les femmes bénéficiant d'une assurance médicale sont surtout celles du milieu urbain (6\% contre $1 \%$ pour le milieu rural), la proportion de femmes sans assurance médicale est élevée dans toutes les régions où elle varie entre $97 \%$ et $100 \%$. De ce lot, se détache la ville d'Abidjan mais avec un pourcentage de $92 \%$, plus bas à peine de quelques points dénotant de la faiblesse de la couverture médicale dans le pays. Les résultats concernant les hommes montrent les mêmes tendances que celles observées chez les femmes : les hommes du milieu urbain (10\%), ceux ayant le niveau d'instruction secondaire et plus (14\%) sont plus couverts par une assurance santé que les hommes du milieu rural (2 $\%)$ ou ceux non instruits (1 \%). Au niveau régional, c'est dans la ville d’Abidjan que les hommes bénéficient le plus d’une assurance médicale (13 $\%)$ tandis que dans toutes les autres régions, cette proportion varie de $2 \%$ au Centre, au Nord-Ouest et au Nord à un maximum de $5 \%$ dans les régions du Centre-Est, du Centre-Nord, du Centre-Ouest et du Sud. Cependant, on constate de faibles écarts entre les différents groupes d’âges, des hommes ou des femmes sans couverture médicale » (l’EDS-MICS 2011-2012).

Dans cette forme de fabrication de rapport, l'assuré a pour objectif la maximisation de son état de santé et la minimisation des coûts de soins prescrits. Dès cet instant, l'assureur veille à ce que l'assuré soit satisfait en lui proposant une bonne prime d'assurance. Or comme susmentionné, sur le marché, on y trouve deux catégories de clients : ceux qui, naturellement, présentent un grand risque de maladie et ceux qui, naturellement, présentent un moindre risque. Les plus petits risques auront tendance à payer des primes 
moins élevées pour une petite couverture tandis que, les usagers aux risques énormes auront tendance à payer des primes plus élevées pour une grande couverture.

Ce constat pose, dans un premier temps, un problème d'anti-sélection du côté de l'assureur. En effet, le souhait de l'assureur est d'avoir des clients potentiellement à haut revenu et présentant un risque moins élevé de maladie ou qui peut se prendre en charge en cas de maladie grave et/ou inguérissable. Les individus à faible dotation économique seront symboliquement laisser pour compte. Exception faite aux «petits » budgets qui sont parfois subventionnés par les employeurs. Par exemple, la mutuelle des fonctionnaires est à taux unique malgré l’hétérogénéité des assureurs. C'est ce que confirme le résultat de l’étude menée par Boyer.S \& al (2000) sur le risque maladie dans les assurances sociales. Selon cette étude, les assurances maladies ne prennent pas en compte la proportionnalité des contributions aux revenus des adhérents et n'accordent pas d'exemption pour les pauvres et les indigents. De l'avis de l'auteur, ce mode de fonctionnement non inclusif contribue à affaiblir la viabilité du système mais aussi et surtout à renforcer davantage l'inégalité dans le processus de couverture médicale. Les entretiens avec les acteurs du système ont permis de savoir que dans l'imaginaire de l'ensemble des services assurance dont le but de leur existence est d'abord lucratif, la prise en charge de la population pauvre et indigente est supposée être du domaine de la politique sociale de l'Etat.

D'autres constats empiriques faits ci-dessus posent souvent un problème d'aléa moral du côté de l'assuré. Par exemple, les entretiens ont permis de comprendre qu'au niveau des risques moraux, les assureurs proposent vivement à leur client de se prévenir contre les endémies, ce qui engendre un coût supplémentaire pour ces derniers. Et si les assurés parviennent à respecter cette condition en cas de maladie, ceux-ci vont consommer plus de soins que prévus. Autant de faits perçus, qui peuvent remettre en cause la viabilité des systèmes d'assurance et l'accès durable de la population aux soins de santé. Certes, ces pratiques d’anti-sélection et aléa moraux appartiennent certainement aux référents socioéconomiques des acteurs qui les portent, mais en se développant, de telles pratiques pourraient limiter significativement l'accès durable des usagers pauvres aux soins de santé. Et la circulation des informations d'acteur en acteur participe au renforcement de cette précarité dans l'accès aux soins. Car « il est mieux mourir que d'être humilier » disent-ils. Les dimensions sociétales, ainsi que la dimension économique de la "chronicisation » des maladies pourraient être interrogées à cet effet. L’étude pourrait questionner prochainement les controverses politiques, idéologiques et économiques, autour des définitions et délimitations du guérissable et de l’inguérissable selon les maisons de micro-assurance. 


\section{2. "L'assurance maladie" comme un "produit d'appel ou d'attraction'” des assurés}

En raison de l'impact de la crise financière marquée surtout par le service de la dette et la chute des cours des produits d'exportation, l'Etat de Côte d'Ivoire a limité sa capacité à financer les services sociaux de base relatifs aux logements, l'éducation, l'adduction d'eau potable et surtout la santé, entrainant ainsi une inégalité dans l'accès aux soins de qualité. En Côte d'Ivoire, la protection sociale est axée principalement sur le secteur formel. En effet, seulement 15\% de la population bénéficie d'une couverture maladie adéquate, $85 \%$ de la population, composé pour la plupart de personnes travaillant dans le secteur informel non structuré, d'agriculteurs, de sans emploi et de personnes indigentes sont exclus d'une couverture maladie adéquate (Lezou, 2006).

Dans ces conditions, l'assurance médicale est positionnée comme une alternative dans la réduction de l'inégalité d'accès aux soins dans la mesure où le traitement de la maladie mobilise des moyens aussi bien physiques que financiers qui peuvent conduire à l'endettement des ménages, les entrainant ainsi dans un cercle vicieux de pauvreté. Mais le constat montre que cela n'est pas le cas. En effet, les entretiens permettent de préciser que le fonctionnement des micro-assurances médicales converge vers un élargissement des inégalités d'accès aux soins de santé de qualité. Cela à cause du double processus observé dans leur mécanisme de mobilisation de clients.

Ainsi, les observations ont permis de savoir que le produit assurance maladie fonctionne, du côté des assureurs, comme un mécanisme d'attraction des usagers. En effet, les maisons d'assurance enquêtées, indiquent de façon unanime que l'assurance maladie constitue un 'produit d'appel' pour elles. C'est par le canal de l'assurance maladie que les assureurs arrivent à avoir plusieurs adhérents. Cela se comprend aisément dans la mesure où les hommes sont prêts à mettre toute leur énergie pour la consolidation de leur état de santé.

La présente étude met en relief la double face de l'assureur. En effet, le produit assurance maladie que l'assureur affiche semble être le produit le plus prisé par la population, alors que, pour l'assureur, ce produit est déficitaire. C'est à cause du caractère déficitaire, du produit ''assurance maladie"' pour l'assureur, à la recherche de gain financier, que celui-ci estime qu'il ne le donne pas uniquement à un usager. Pendant que l'assurance maladie est le produit le plus affectionné par la population, elle est vue par l'assureur comme un produit déficitaire. Ce regard différent de part et d'autre ne peut qu'affecter le taux de couverture maladie.

Donc, l'usager qui souscrit au produit 'assurance maladie"' est incité à impérativement souscrire à d'autres produits d'assurance (incendie, 
automobile, vie, etc.) pour bénéficier pleinement de l’assurance maladie. Sur la base des entretiens, les assurés ne sont pas informés préalablement du caractère " "produit d'appel”' que les assureurs attribuent au produit assurance maladie. A cet effet, l'enquête a permis de savoir que les différents produits proposés par les maisons d'assurance sont : l'assurance santé, multirisque des artisans et commerçants, assurance automobile, multirisque habitation, assurance construction, assurance caution, assurance individuelle, accidents corporels, tous-risques informatique, assurance voyage et déplacement, assurance vie etc. Après souscription, ces différents produits sont proposés aux clients afin qu'il bénéficie pleinement des fruits de sa cotisation. C'est après souscription que l'usager est informé et contraint à souscrire au second produit pour bénéficier de l'assurance maladie. Cet enquêté, âgé de 37 ans, responsable d'une assurance affirme que :

«Nous sommes des sociétés commerciales et nous sommes à la recherche du profit, nous savons très bien que la majorité de la population n'a pas de couverture maladie et nous sommes sensibles à cette situation, mais nous ne pouvons pas fixer des primes ou se limiter au seul produit 'assurance maladie' 'qui mettre en difficulté financière notre petite société ; déjà que l'assurance maladie est très déficitaire ».

Ces propos mettent en lumière deux réalités. Premièrement, cette attitude des petits fournisseurs de prestation de service assurance produira dans un futur proche ou lointain un manque de confiance entre assureurs et assurés. Sans confiance, la viabilité du système dans le temps et l'espace ne sera pas possible, puisque le taux d'adhésion sera faible et le taux de déperdition des assurés augmentera d’année en année. C’est pour cette raison Criel, Pierre.B. \& alii (2006) affirment que l'adhésion à un système et la viabilité de celui-ci est lié à trois éléments majeurs : la capacité du ménage à payer les contributions (i), la qualité de l'offre (ii) et la confiance au système (iii).

Deuxièmement, les individus à faible dotation économique observeront un jour ou l'autre des limites d'accès à la santé. Car un usager peut avoir sa prime annuelle d'assurance maladie et ne pas avoir la prime ou n'ayant pas besoin du produit que lui ''impose', l'assureur. Ce comportement caché des “'petits'” assureurs s'explique par le fait qu'une seule hospitalisation ou la maladie d'un seul usager peut entrainer la consommation totale de la prime annuelle versée par l'usager. Or l'assureur cherche à maximiser son profit pour ne pas que son 'grenier" s'effondre. Car disent-ils, ce sont les primes des usagers qui constituent les bois de soutènement du 'grenier".

Dans ces conditions, au lieu de lutter contre la pauvreté et améliorer l'accès aux soins, comme le soutient Dussault \& alii (2006), les microassurances maladie pourraient renforcer l’inégalité dans l'accès aux soins et 
instaurer une sorte de pauvreté de ses membres assurés. Dans l’imaginaire des assureurs ou fournisseurs de prestations, l'élargissement de la couverture des prestations de service suffit pour influencer l'attractivité des produits qu'ils proposent. Les résultats de l'étude permettent de remettre en cause ce mode de fonctionnement très sélectif dans la mesure où elle influence négativement non seulement la confiance qui doit lier les assureurs aux assurés mais aussi exclut la population à faible revenu financier de la couverture du risque face à la maladie.

Cependant, ce processus sélectif animé par les assureurs, n’est pas de nature à produire un accès durable aux soins de santé, surtout pour la population pauvre. Le consentement à cotiser pour l'assurance maladie n'est pas le fait du hasard mais plutôt expliqués par la rationalité économique de l'individu. C'est pour cette raison que Bruno.G (2006) met en exergue la fonction d'utilité dans le processus d'adhésion à un système d'assurance maladie. Ainsi nous convenons avec Bruno-G-(2006) que c'est le montant de la cotisation que doit payer l'assuré qui est en jeu (taux unitaire, modalité d’adhésion) par rapport aux service apporté.

\section{Conclusion}

Les faits observés, sur le comportement de deux acteurs de l'assurance maladie, ont permis de déboucher sur le résultat selon lequel la nature des relations assureurs (système assurance) et assurés (population) pourrait limiter l'accès de la population aux soins de santé de qualité et fragiliser aussi la viabilité financière du système d'assurance maladie.

En effet, le risque aléa moral, l'anti-sélection et divinisation de l'usager produit à la fois par la population et le système assurance constituent des facteurs de non viabilité du système. Conséquemment, cela limite l'accès durable de la population aux services de santé de qualité. Par exemple, les entretiens ont permis de comprendre qu'au niveau des risques moraux (phycologiques), les assureurs imposent à leur client de se prévenir contre les endémies, ce qui engendre un coût supplémentaire pour ces derniers. Et si les assurés parviennent à respecter cette condition en cas de maladie, ceux-ci vont consommer plus de soins que prévus.

\section{References:}

Bruno. G (2006)., Assurance maladie en Afrique francophone. Banque Mondiale, pp. 331-337

Boyer.S. \& al (2000)., Le risque maladie dans les assurances sociale : bilan et perspective dans les PVD. Centre de recherche, d'étude et de documentation en économie de la santé (CREDES)

Drechsle D \& Johannes (2005)., l'assurance maladie privée dans les pays en développement, une solution pour les pauvres ? Repères n¹1, Aout. 
De Sardan J-P O, 2000, Rendre compte des points de vue des acteurs : principes méthodologiques de l'enquête de terrain en Sciences sociales, in Les enquêtes participatives en débat. Ambitions, pratiques et enjeux ? Lavigne - Delville Ph., et autres (éds.), Paris, GRET - KARTHALA ICRA, pp. 421-449

Dussault \& alii (2006)., l'assurance maladie en Afrique francophone: améliorer l'accès aux soins et lutter contre la pauvreté ; banque mondiale N'guessan. \& Bouaffon.V. (2006)., contribution des ménages ruraux aux financements de l'assurance maladie universelle en Côte d'Ivoire: une analyse à partir du modèle Tobit censure; conférence AED, Paris, septembre 2006.

Lezou.P. (2006)., le système de protection sociale et la réalité du droit de la santé, Mémoire de DESS, Université Cocody.

Yves Eggli, \& alii (2006)., Analyse des prestations prises en charge par l'assurance maladie obligatoire des soins, Cadre conceptuel et étude de faisabilité centrée sur trois pathologies : cancer, diabète et affections mentales. Rapports d'experts et de recherche pour l'assurance-maladie et accidents ; Office fédérale de la santé publique(OFSP) 